\title{
ATOMIC AND NONATOMIC MEASURES
}

\author{
ROY A. JOHNSON
}

\begin{abstract}
Elementary properties of atomic and nonatomic measures are investigated. In addition, it is shown that every measure can be written as the sum of a purely atomic measure and a nonatomic measure. Conditions are given under which this decomposition is unique.
\end{abstract}

1. Atomic and nonatomic measures. Suppose $\mu$ is a (nonnegative, countably additive) measure on the $\sigma$-ring $S$. A set $E \in S$ will be called an atom for $\mu$ if (1) $\mu(E)>0$ and (2) given $F \in \delta$, either $\mu(E \cap F)$ or $\mu(E-F)$ is 0 . We notice that if $E$ is an atom for $\mu$ and $\mu(E \cap F)>0$, then $E \cap F$ is also an atom for $\mu$. We shall say that $\mu$ is purely atomic or simply atomic if every measurable set of positive measure contains an atom. We shall say that $\mu$ is nonatomic if there are no atoms for $\mu$. This means that every measurable set of positive measure can be split into two disjoint measurable sets, each having positive measure. Clearly the zero measure is the only measure which is both purely atomic and nonatomic.

The purpose of this paper is to investigate elementary properties of these measures and the relations between these measures. For example, as is well known, a $\sigma$-finite measure can be written uniquely as the sum of a purely atomic measure and a nonatomic measure. But this uniqueness is not automatic in the general case. Much of our effort will be devoted to studying relations between not necessarily $\sigma$-finite measures.

THEOREM 1.1. Suppose $\nu$ and $\lambda$ are purely atomic measures on $s$. Then so are $\nu+\lambda$ and $\nu \bigvee \lambda$, where $\nu \bigvee \lambda$ is the smallest measure greater than or equal to $\nu$ and $\lambda$.

Proof. Let $\mu=\nu+\lambda$ or let $\mu=\nu \bigvee \lambda$, since the same proof works in both cases. Suppose $\mu(E)>0$. Then $\nu(E)>0$ or $\lambda(E)>0$. Let us suppose $\nu(E)>0$. Since $\nu$ is purely atomic, there exists a $\nu$-atom $F$ such that $F \subset E$. If $\lambda(F)=0$, then $F$ is clearly an atom for $\mu$. On the other hand, if $\lambda(F)>0$, then there exists a $\lambda$-atom $G$ such that $G \subset F$. Of course $\mu(G)>0$. If $G$ is an atom for $\mu$, then we are done. Otherwise,

Received by the editors July 18, 1969.

AMS Subject Classifications. Primary 2813.

Key Words and Phrases. Atom, purely atomic measure, atomic measure, nonatomic measure, sigma-finite measure, semifinite measure. 
there exists $H \in S$ such that $\mu(G \cap H)>0$ and $\mu(G-H)>0$. In that case, $G \cap H$ will serve as the required atom for $\mu$. (Indeed, $G-H$ would work also.)

THEOREM 1.2. Suppose $\left\{\mu_{\alpha}\right\}$ is a family of nonatomic measures and that $\mu=\sum \mu_{\alpha}$ or $\mu=V_{\mu_{\alpha}}$, the smallest measure greater than or equal to each $\mu_{\alpha}$. Then $\mu$ is nonatomic also.

Proof. Suppose $\mu(E)>0$. Then there exists $\mu_{\alpha}$ such that $\mu_{\alpha}(E)>0$. Since $\mu_{\alpha}$ is nonatomic, there exists $F$ such that $\mu_{\alpha}(E \cap F)>0$ and $\mu_{\alpha}(E-F)>0$. But then $\mu(E \cap F)>0$ and $\mu(E-F)>0$, so that $\mu$ is nonatomic.

It would be pleasant if the analogue of Theorem 1.2 held for atomic measures, but this is not the case. Example 1.3 shows that $\sum \mu_{\alpha}$ and $V \mu_{\alpha}$ can be nonatomic, even though each $\mu_{\alpha}$ is atomic.

If $\left\{\mu_{n}\right\}$ is a sequence of atomic measures, I do not know if $\sum \mu_{n}$ or $V \mu_{n}$ is atomic.

EXAMPLE 1.3. Let $X$ be a compact Hausdorff space such that each singleton set fails to be a $G_{\delta}$. Let $\delta$ be the $\sigma$-ring generated by compact $G_{\delta}$ subsets of $X$. For each $x \in X$, we define $\mu_{x}$ by $\mu_{x}(E)=1$ if $x \in E$ and 0 otherwise. It is clear that each $\mu_{x}$ is atomic. But if $\mu$ is defined by $\mu(E)=0$ if $E$ is empty and $\infty$ otherwise, then $\mu$ is nonatomic, even though $\mu=\sum\left\{\mu_{x}: x \in X\right\}$ and $\mu=V\left\{\mu_{x}: x \in X\right\}$.

We say that $\nu$ is absolutely continuous with respect to $\mu$, denoted $\nu \ll \mu$, if $\nu(F)=0$ whenever $\mu(F)=0$.

THEOREM 1.4. If $E$ is an atom for $\mu$ and $\nu \ll \mu$, then either $\nu(E)=0$ or $E$ is an atom for $\nu$.

Proof. Suppose $E$ is an atom for $\mu$. Then, given $F \in \mathcal{S}$, either $\mu(E \cap F)=0$ or $\mu(E-F)=0$. Hence, at least one of $\nu(E \cap F)$ or $\nu(E-F)$ is equal to 0 , and this proves the result.

If $\mu$ and $\nu$ are measures on $\delta$, we shall say that $\nu$ is $S$-singular with respect to $\mu$, denoted $\nu S \mu$, if given $E \in S$, there exists $F \in S, F \subset E$, such that $\nu(E)=\nu(F)$ and $\mu(F)=0$ [3, p. 628]. Equivalently, $\nu(E)=\sup \{\nu(E \cap F): \mu(F)=0\}$.

We already know that the sum of two purely atomic measures or two nonatomic measures is a measure of the same type. But if the sum of two measures, $\nu$ and $\lambda$, is atomic [nonatomic], does it follow that $\nu$ and $\lambda$ are themselves atomic [resp., nonatomic]? Examples 2.9 and 2.10 will show that the answer is no, but the following holds:

THEOREM 1.5. If $\mu=\nu+\lambda$, where $\nu S \lambda$, then $\nu$ is atomic or nonatomic if $\mu$ has that property. 
Proof. Suppose $\nu(E)>0$. Since $\nu S \lambda$, there exists $F \in \mathcal{S}, F \subset E$, such that $\nu(F)>0$ and such that $\lambda(F)=0$. Of course $\mu(F)>0$. Now if $\mu$ is atomic, there exists a $\mu$-atom $G \subset F$. Since $\nu \ll \mu$, either $\nu(G)=0$ (impossible) or $G$ is an atom for $\nu$, as was to be shown. On the other hand if $\mu$ is nonatomic, then there exists $G \in \mathcal{S}$ such that $\mu(F \cap G)>0$ and $\mu(F-G)>0$. Necessarily, $\nu(F \cap G)>0$ and $\nu(F-G)>0$, so that $\nu$ is nonatomic.

The following theorem may appear somewhat artificial, but it is quite useful in proving the uniqueness part of Theorem 2.1.

THEOREM 1.6. If $\nu \ll \lambda+\mu$ and $\mu S \lambda$, where $\nu$ is purely atomic and $\mu$ is nonatomic, then $\mu S \nu$. Hence, if $\mu$ is nonatomic, $\nu$ is atomic, and $\nu \ll \mu$, then $\mu S \nu$.

Proof. By the Lebesgue decomposition theorem [5, 1.1], we may write $\mu=\mu_{1}+\mu_{2}$, where $\mu_{1} \ll\left\langle, \mu_{2} S \nu\right.$, and $\mu_{1} S \mu_{2}$. In view of Theorem $1.5, \mu_{1}$ and $\mu_{2}$ are nonatomic. It suffices to show that $\mu_{1}=0$. Suppose, to the contrary that $\mu_{1}(E)>0$. Then $\nu(E)>0$. Since $\mu_{1} S \mu_{2}$ and $\mu S \lambda$, we may assume that $\mu_{2}(E)=0$ and that $\lambda(E)=0$. Since $\nu$ is purely atomic and $\nu(E)>0$, there exists a $\nu$-atom $F \subset E$. Necessarily, $(\mu+\lambda)(F)>0$; whence it follows that $\mu_{1}(F)>0$. Hence, by Theorem 1.4 we see that $F$ is an atom for $\mu_{1}$, which is impossible. Hence, $\mu_{1}=0$, as was to be shown.

2. A decomposition. Suppose $\mu$ is a measure on $\delta$ and that $\mathfrak{T}$ is a subfamily of $S$ such that 97 is closed under countable unions. Of course, $\varnothing \in \mathfrak{I}$. If, for each $E \in S$, we define

$$
\mu_{1}(E)=\sup \{\mu(E \cap M): M \in \mathscr{N}\}
$$

and

$$
\mu_{2}(E)=\sup \left\{\mu(E \cap N): \mu_{1}(N)=0\right\},
$$

then it is easy to see that $\mu_{1}$ and $\mu_{2}$ are measures on $\delta$ such that (1) $\mu=\mu_{1}+\mu_{2}$, (2) $\mu_{2} S \mu_{1}$ and (3) $\mu_{1} S \mu_{2}$ (cf. [3, 628-629]). To show, for example, that $\mu_{1} S \mu_{2}$, we observe that if $\mu_{1}(N)=0$, then $\mu(N \cap M)$ $=0$ for all $M \in \mathscr{T}\left(\right.$ and hence $\mu_{2}(M)=0$ for all $M \in \mathscr{T}$. It follows that $\mu_{1} S \mu_{2}$. We now show that every measure can be written as the sum of a purely atomic measure and a nonatomic measure.

TheOREM 2.1. If $\mu$ is any measure on $\delta$, then there exist measures $\mu_{1}$ and $\mu_{2}$ such that $\mu=\mu_{1}+\mu_{2}$, where $\mu_{1}$ is purely atomic and $\mu_{2}$ is nonatomic. We may choose $\mu_{1}$ and $\mu_{2}$ such that $\mu_{1} S \mu_{2}$ and $\mu_{2} S \mu_{1}$, and under these conditions $\mu_{1}$ and $\mu_{2}$ are unique. 
Proof. Let $\mathfrak{T}$ be the family of all countable unions of $\mu$-atoms. For each $E \in S$, let

$$
\mu_{1}(E)=\sup \{\mu(E \cap M): M \in \mathbb{T}\}
$$

and

$$
\mu_{2}(E)=\sup \left\{\mu(E \cap N): \mu_{1}(N)=0\right\} .
$$

Then $\mu=\mu_{1}+\mu_{2}$, where $\mu_{1} S \mu_{2}$ and $\mu_{2} S \mu_{1}$. We show that $\mu_{1}$ is purely atomic. Suppose $E \in S$. If $\mu_{1}(E)>0$, then $\mu(E \cap M)>0$ for some $M \in \mathfrak{T}$. If $M=\mathrm{U} M_{n}$, where each $M_{n}$ is a $\mu$-atom, then $\mu\left(E \cap M_{n}\right)>0$ for some $\mu$-atom $M_{n}$. Since $E \cap M_{n}$ is a $\mu$-atom such that $\mu_{1}\left(E \cap M_{n}\right)$ $>0$ and since $\mu_{1} \leqq \mu$, we have $E \cap M_{n}$ is a $\mu_{1}$-atom contained in $E$.

To show that $\mu_{2}$ is nonatomic, suppose that $\mu_{2}(E)>0$. Then $\mu(E \cap N)>0$ for some $N$ such that $\mu_{1}(N)=0$. We know that $E \cap N$ is not a $\mu$-atom; otherwise $\mu_{1}(E \cap N)>0$. Now since $\mu(E \cap N)>0$ and since $E \cap N$ is not a $\mu$-atom, there exists $F \in \mathcal{S}$ such that $\mu(E \cap N \cap F)$ $>0$ and $\mu((E \cap N)-F)>0$. Necessarily, $\mu_{2}(E \cap F)>0$ and $\mu_{2}(E-F)$ $>0$, which shows that $\mu_{2}$ is nonatomic.

We now show uniqueness. Suppose that $\mu$ can also be written as $\nu_{1}+\nu_{2}$, where $\nu_{1}$ is purely atomic, $\nu_{2}$ is nonatomic, $\nu_{1} S \nu_{2}$, and $\nu_{2} S \nu_{1}$. Since $\nu_{1} \leqq \mu_{1}+\mu_{2}$ and $\mu_{2} S \mu_{1}$, we have $\mu_{2} S \nu_{1}$ by Theorem 1.6. Then, since $\mu_{2} \leqq \nu_{1}+\nu_{2}$ and $\mu_{2} S \nu_{1}$, we have $\mu_{2} \leqq \nu_{2}$ [3, p. 630]. Similarly, $\nu_{2} \leqq \mu_{2}$. Then $\nu_{1} \leqq \mu_{1}+\mu_{2}$ and $\nu_{1} S \mu_{2}$, so that $\nu_{1} \leqq \mu_{1}$. Similarly, $\mu_{1} \leqq \nu_{1}$, and we are done.

We notice in the proof of Theorem 2.1, that if $\mu$ is atomic and $E \in S$, then there exists a countable collection of atoms $M_{n}$ such that $\mu(E)=\mu\left(E \cap \bigcup M_{n}\right)$. Letting

$$
E_{n}=\left[M_{n}-\left(M_{1} \cup \ldots \cup M_{n-1}\right)\right] \cap E
$$

and disregarding those $E_{n}$ 's, if any, having zero measure, we have the following:

THEOREM 2.2. If $\mu$ is purely atomic and $\mu(E)>0$, then there exists a countable disjoint collection of atoms $E_{k} \subset E$ such that $\mu(E)=\mu\left(U E_{k}\right)$.

Theorem 2.3. If $\mu$ is atomic, $\nu$ is nonatomic, and $\nu \ll \mu$, then $\mu S \nu$.

Proof. Suppose $\mu(E)>0$. By Theorem 2.2, there exists a countable disjoint collection of atoms $E_{k} \subset E$ such that $\mu(E)=\mu\left(U E_{k}\right)$. By Theorem 1.4 we see that $\nu\left(E_{k}\right)=0$ for each $k$, so that $\nu\left(U E_{k}\right)=0$.

Theorem 2.4. Suppose $\nu \ll \mu$, where $\mu$ is $\sigma$-finite. If $\mu$ is atomic, then so is $\nu$. If $\mu$ is nonatomic, then so is $\nu$. Hence, if $\nu$ is nonzero and atomic, then $\mu$ has an atom. 
PROOF. In view of Theorem 2.1, we may write $\nu=\nu_{1}+\nu_{2}$, where $\nu_{1}$ is atomic and $\nu_{2}$ is nonatomic. Suppose $\mu$ is atomic. Then since $\nu_{2} \ll \mu$, we have $\mu S \nu_{2}$ by Theorem 2.3. Since $\mu$ is $\sigma$-finite, we have $\nu_{2} S \mu$ $[3,3.2]$, and hence $\nu_{2}=0[3$, p. 629]. On the other hand, suppose $\mu$ is nonatomic. Then since $\nu_{1} \ll \mu$, we have $\mu S \nu_{1}$ by Theorem 1.6. As before, we have $\nu_{1} S \mu$, so that $\nu_{1}=0$.

THEOREM 2.5. If $\mu$ is $\sigma$-finite and atomic [nonatomic] and $\nu$ is nonatomic [resp., atomic], then $\nu S \mu$.

ProOF. By the Lebesgue decomposition theorem [5, 1.1], we may write $\nu=\nu_{1}+\nu_{2}$, where $\nu_{1} \ll \mu, \nu_{2} S \mu$, and $\nu_{1} S \nu_{2}$. By Theorem 1.5 we have $\nu_{1}$ is atomic [nonatomic], and by Theorem 2.4 we have $\nu_{1}$ is nonatomic [atomic]. Hence, $\nu_{1}=0$, and we are done.

CoROllary 2.6. If $\mu$ is a $\sigma$-finite measure on $\mathcal{S}$, then there exist unique measures $\mu_{1}$ and $\mu_{2}$ such that $\mu=\mu_{1}+\mu_{2}$ and such that $\mu_{1}$ is purely atomic and $\mu_{2}$ is nonatomic.

Proof. Such $\mu_{1}$ and $\mu_{2}$ exist by Theorem 2.1. Then by Theorem 2.5 we have $\mu_{1} S \mu_{2}$ and $\mu_{2} S \mu_{1}$, so that uniqueness follows.

A measure $\mu$ on the $\sigma$-ring $\delta$ will be called semifinite if every measurable set of infinite measure contains a measurable set of finite, positive measure (cf. [1, Exercise 25.9]). We notice that the atoms, if any, for a semifinite measure must be of finite measure.

THEOREM 2.7. Suppose $\left\{\mu_{\alpha}\right\}$ is a family of atomic measures and that $\mu=V \mu_{\alpha}$, the smallest measure greater than or equal to each $\mu_{\alpha}$. If $\mu$ is semifinite, then it is atomic.

Proof. Suppose $\mu(E)>0$. Since $\mu$ is semifinite, we may assume that $\mu(E)<\infty$. Consider $\nu_{\alpha}$ and $\nu$, the restrictions of $\mu_{\alpha}$ and $\mu$ to $\delta \cap E$. We have $\nu_{\alpha}(E)>0$ for some $\alpha$, and since $\nu_{\alpha} \leqq \nu$, we see that $\nu$ has an atom $F$ by Theorem 2.4. Clearly, $F$ is a $\mu$-atom contained in $E$.

THEOREM 2.8. Suppose $\left\{\mu_{\alpha}\right\}$ is a family of atomic measures. If $\mu=\sum \mu_{\alpha}$ and $\mu$ is semifinite, then $\mu$ is atomic also.

Proof. Immediate from Theorems 1.1 and 2.7.

Can the $\sigma$-finiteness requirement on $\mu$ in Theorems 2.4 and 2.5 be weakened to semifiniteness? The following examples show that the answer is no.

EXAMPLE 2.9. Let $\mu$ and $\nu$ be counting measure and Lebesgue measure, resp., on the Borel sets of the unit interval. It is easy to see that $\nu \leqq \mu$, even though $\nu$ is nonatomic and $\mu$ is atomic. 
Example 2.10. Let $X$ be an uncountable set, $Y$ be the unit interval, and $m$ be Lebesgue measure on the Borel sets $B$ of the unit interval. As usual, $E_{x}$ denotes $\{y:(x, y) \in E\}$. Let $S$ be the class of all $E \subset X \times Y$ such that $E_{x} \in \Theta$ for all $x \in X$ and such that either $\left\{x: E_{x} \neq \varnothing\right\}$ or $\left\{x: E_{x} \neq Y\right\}$ is countable. We define measures $\mu$ and $\nu$ on $S$ as follows: Let $\nu(E)=0$ if $\left\{x: E_{x} \neq \varnothing\right\}$ is countable and 1 otherwise. Let $\mu(E)$ $=\sum\left\{m\left(E_{x}\right): x \in X\right\}$. It is clear that $\mu$ is nonatomic, $\nu$ is atomic, and $\nu \leqq \mu$.

It is possible for a nonzero atomic measure to be zero on singleton sets, e.g., Example 2.10. However, there exists a large class of $\sigma$-rings $\mathcal{S}$ such that a nonzero atomic measure on $\mathcal{S}$ must be nonzero on at least one singleton set. For example, consider any topological Hausdorff space such that each finite measure on the Borel sets is a regular Borel measure. (Any compact metric space will do.) If a finite measure on the Borel sets of such a space is zero on singleton sets, then the measure is nonatomic [4, 2.IV]. Then in view of Theorem 2.11, any measure on the Borel sets of such a space and vanishing on singleton sets will be nonatomic.

THEOREM 2.11. If $\mu$ has an atom, then there exists a finite, nonzero atomic measure $\nu$ such that $\nu \leqq \mu$.

Proof. Suppose $F$ is an atom for $\mu$. If $\mu(F)<\infty$, let $\nu(E)=\mu(E \cap F)$ for all $E \in$ s. If $\mu(F)=\infty$, let $\nu(E)=0$ or 1 as $\mu(E \cap F)=0$ or $\infty$.

\section{REFERENCES}

1. S. K. Berberian, Measure and integration, Macmillan, New York, 1965. MR 32 $\# 1315$.

2. P. R. Halmos, Measure theory, Van Nostrand, Princeton, N. J., 1950. MR 11 $\# 504$.

3. R. A. Johnson, On the Lebesgue decomposition theorem, Proc. Amer. Math. Soc. 18 (1967), 628-632. MR 36 \#1602.

4. J. D. Knowles, On the existence of non-atomic measures, Mathematika 14 (1967), 62-67. MR 35 \#5568.

5. N. Y. Luther, Lebesgue decomposition and weakly Borel measures, Duke Math. J. 35 (1968), 601-615. MR 37 \#5346.

Washington State University, Pullman, Washington 99163 